\title{
Erratum to: Tracheobronchomalacia in children: review of diagnosis and definition
}

\section{Judith Zhi Yie Tan • Michael Ditchfield • Nicholas Freezer}

Published online: 10 October 2012

(C) Springer-Verlag Berlin Heidelberg 2012

\section{Erratum to: Pediatr Radiol}

\section{DOI 10.1007/s00247-012-2367-5}

Figure 1 caption contains an error. The corrected caption appears below.

Fig. 1 Cine fluoroscopy with oral contrast agent. a, b Images demonstrate posterior indentation of the oesophagus (asterisk) in a child with a confirmed double aortic arch. There is a marked change in tracheal calibre (arrows) in expiration (b) relative to inspiration (a)

The online version of the original article can be found at http://dx.doi.org/ 10.1007/s00247-012-2367-5.

J. Z. Y. Tan $(\bowtie) \cdot$ M. Ditchfield

Department of Diagnostic Imaging,

Monash Children's, Southern Health,

246 Clayton Road,

Clayton 3168 Victoria, Australia

e-mail: zhi_yie@yahoo.com.au

M. Ditchfield $\cdot$ N. Freezer

Monash University,

Clayton Road,

Clayton 3168 Victoria, Australia

N. Freezer

Paediatric Respiratory and Sleep Medicine,

Monash Children's, Southern Health,

246 Clayton Road,

Clayton 3168 Victoria, Australia 